\title{
Acute effects of ingestion of a novel whey-derived extract on vascular endothelial function in overweight, middle-aged men and women
}

\author{
Kevin D. Ballard ${ }^{1,2 *}$, Brian R. Kupchak ${ }^{1}$, Brittanie M. Volk ${ }^{1}$, Eunice Mah $^{2}$, Aida Shkreta ${ }^{3}$, \\ Cary Liptak $^{3}$, Adam S. Ptolemy ${ }^{3}$, Mark S. Kellogg ${ }^{3}$, Richard S. Bruno ${ }^{2}$, Richard L. Seip ${ }^{4}$, Carl M. Maresh ${ }^{1}$, \\ William J. Kraemer ${ }^{1}$ and Jeff S. Volek ${ }^{1}$ \\ ${ }^{1}$ Department of Kinesiology, University of Connecticut, 2095 Hillside Road, Unit 1110, Storrs, CT 06269, USA \\ ${ }^{2}$ Department of Nutritional Sciences, University of Connecticut, 3624 Horsebarn Road Extension, Roy E. Jones Building, \\ Unit 4017, Storrs, CT 06269, USA \\ ${ }^{3}$ Department of Laboratory Medicine, Children's Hospital Boston, Boston, MA 02115, USA \\ ${ }^{4}$ Genetics Research Center, Hartford Hospital, Hartford, CT 06102, USA
}

(Submitted 1 November 2011 - Final revision received 16 April 2012 - Accepted 24 April 2012 - First published online 13 June 2012)

\begin{abstract}
Whey protein intake reduces CVD risk, but little is known whether whey-derived bioactive peptides regulate vascular endothelial function (VEF). We determined the impact of a whey-derived extract (NOP-47) on VEF in individuals with an increased cardiovascular risk profile. Men and women with impaired brachial artery flow-mediated dilation (FMD) ( $n$ 21, age 55 (SEM 1.3) years, BMI 27.8 (SEM 0.6) kg/m ${ }^{2}$, FMD 3.7 (SEM 0.4) \%) completed a randomised, cross-over study to examine whether ingestion of NOP-47 (5g) improves postprandial VEF. Brachial artery FMD, plasma amino acids, insulin, and endothelium-derived vasodilators and vasoconstrictors were measured for $2 \mathrm{~h}$ after ingestion of NOP-47 or placebo. Acute NOP-47 ingestion increased FMD at 30 min (4.6 (SEM 0.5) \%) and 120 min (5.1 (SEM 0.5) \%) post-ingestion $(P<0.05$, time $\times$ trial interaction), and FMD responses at 120 min were significantly greater in the NOP-47 trial compared with placebo ( 4.3 (SEM 0.5$) \%$ ). Plasma amino acids increased at $30 \mathrm{~min}$ following NOP-47 ingestion $(P<0.05)$. Serum insulin increased at 15,30 and $60 \mathrm{~min}(P<0 \cdot 001)$ following NOP-47 ingestion. No changes were observed between the trials for plasma NO and prostacyclin metabolites or endothelin-1. Ingestion of a rapidly absorbed extract derived from whey protein improved endothelium-dependent dilation in older adults by a mechanism independent of changes in circulating vasoactive compounds. Future investigation is warranted in individuals at an increased CVD risk to further elucidate potential health benefits and the underlying mechanisms of extracts derived from whey.
\end{abstract}

Key words: Vascular endothelial function: Flow-mediated dilation: Nitric oxide: Amino acids: Whey protein

CVD accounts for approximately 800000 deaths annually and is the leading cause of morbidity and mortality in the USA ${ }^{(1)}$. The risk of CVD is substantially increased by endothelial dysfunction $^{(2)}$, a process mediated through alterations in vasomotor tone, platelet adhesion and thrombosis, all of which are regulated by vasoactive compounds ${ }^{(3)}$. Suppression of endothelial functions is an early event in the progression of atherosclerosis ${ }^{(4)}$ and therefore represents an important target for assessment in order to manage the risk for developing CVD.

Endothelium-dependent vasodilation can be assessed 'non-invasively' in the peripheral circulation by brachial artery flow-mediated dilation (FMD) ${ }^{(5)}$. Assessment of endothelial function using FMD has been suggested to represent a functional surrogate for $\mathrm{NO}^{\circ}$ bioavailability in humans ${ }^{(6)}$, although recent findings have demonstrated that $\mathrm{NO}^{\circ}$ is not obligatory for conduit artery FMD in healthy adults ${ }^{(7)}$. Indeed, FMD responses are related to coronary artery endothelial function ${ }^{(8)}$ as well as risk factors for CVD and mortality due to $\mathrm{CVD}^{(9)}$. Thus, impaired FMD responses are regarded as an early manifestation in the progression of $\mathrm{CVD}^{(10)}$.

Ageing is an inevitable biological process that represents a major, independent risk factor for CVD morbidity and mortality ${ }^{(2)}$. Impaired vascular endothelial function, as demonstrated by reduced endothelium-dependent dilation, has been reported in otherwise healthy middle-aged and older adults $^{(11,12)}$. Biosynthesis and/or bioavailability of $\mathrm{NO}^{\circ}$ is impaired in healthy older humans and represents a critical mechanism underlying ageing-mediated endothelial

Abbreviations: \%FMD, percentage of flow-mediated dilation; ACE, angiotensin-converting enzyme; ET-1, endothelin-1; FMD, flow-mediated dilation; PRE, pre-ingestion; $\mathrm{SR}_{\mathrm{AUC}}$, area under the shear rate curve; UPLC, ultra-performance liquid chromatography. 
dysfunction $^{(13)}$. Therefore, interventions that improve $\mathrm{NO}^{\circ}$ homeostasis would not only be expected to improve endothelial function, but also the risk of CVD-related morbidity and mortality.

Bioactive peptides derived from food, especially dairy products, have been shown to exert numerous bioactivities including decreasing blood pressure ${ }^{(14,15)}$ and improving endothelial function ${ }^{(16)}$. Milk-derived peptides also inhibit angiotensin-converting enzyme (ACE) activity in vitro ${ }^{(17,18)}$. That chronic whey consumption in humans reduces blood pressure $^{(18,19)}$ and protects against vascular dysfunction ${ }^{(19)}$ has prompted studies to examine whether its acute ingestion can regulate postprandial responses leading to impaired vascular function. Our prior work demonstrated that acute ingestion of an extract isolated from a whey protein hydrolysate (NOP-47) improved $\mathrm{NO}^{\circ}$-dependent and -independent vascular responses ${ }^{(20)}$. Because our prior study was limited to young adults having a low risk for $\mathrm{CVD}^{(20)}$, the present study aimed to examine whether acute ingestion of NOP-47 would improve postprandial vascular function in older adults with confirmed impaired vascular endothelial function. To this end, we conducted a randomised, placebo-controlled, cross-over study to examine the effect of acute NOP-47 ingestion on FMD responses and biomarkers of vascular function during a $2 \mathrm{~h}$ postprandial period.

\section{Methods \\ Study design}

The present study was conducted according to the guidelines laid down in the Declaration of Helsinki and all procedures involving human subjects were approved by the Institutional Review Board at the University of Connecticut. Written informed consent was obtained from all subjects. A randomised, double-blinded, placebo-controlled, cross-over study design with a washout period was conducted. Each participant reported to the laboratory for three separate visits. Participants participated in one screening visit and, if they qualified, two acute vascular testing trials in which they ingested either a whey-derived extract (NOP-47; Table 1) or placebo. To ensure standardisation between the acute vascular testing trials, participants were instructed to maintain their current level of physical activity during the study period. Additionally, participants recorded their dietary intake $1 \mathrm{~d}$ before the first acute trial and replicated this diet record before the second acute trial. In order to reduce confounding effects on experimental outcomes, participants were instructed to fast and avoid caffeine for $12 \mathrm{~h}$, avoid alcohol, strenuous exercise, aspirin and over-the-counter medications for $24 \mathrm{~h}$, avoid any vitamins and supplements for $72 \mathrm{~h}^{(21)}$, and to consume 1 litre of water the night before and $480 \mathrm{ml}$ the morning of each trial to ensure adequate hydration.

\section{Participants}

Healthy, non-smoking men ( $n$ 11) and postmenopausal women ( $n$ 10) were recruited on the basis of age (45-65 years), BMI
Table 1. Amino acid composition of the whey extract (NOP-47)

\begin{tabular}{lcc}
\hline Amino acids & $\mathrm{g} / 100 \mathrm{~g}$ powder & $5 \mathrm{~g} \mathrm{NOP}-47^{*}$ \\
\hline Trp & 1.33 & 0.07 \\
Cystine & 0.52 & 0.03 \\
Met & 5.05 & 0.25 \\
Asp & 5.46 & 0.27 \\
Thr & 10.74 & 0.54 \\
Ser & 4.29 & 0.21 \\
Glu & 8.21 & 0.41 \\
Pro & 2.30 & 0.12 \\
Gly & 1.18 & 0.06 \\
Ala & 6.86 & 0.34 \\
Val & 7.31 & 0.37 \\
Ile & 5.69 & 0.28 \\
Leu & 22.40 & 1.12 \\
Tyr & 2.31 & 0.12 \\
Phe & 4.41 & 0.22 \\
Lys & 5.79 & 0.29 \\
His & 1.70 & 0.09 \\
Arg & 1.35 & 0.07 \\
\hline
\end{tabular}

* The dose used in the present study was $5 \mathrm{~g}$ of NOP-47 powder mixed with artificial sweetener in $300 \mathrm{ml}$ water.

$\left(25-40 \mathrm{~kg} / \mathrm{m}^{2}\right)$, fasting total cholesterol $(<3200 \mathrm{mg} / \mathrm{l})$ and glucose $(<1200 \mathrm{mg} / \mathrm{l})$ and resting blood pressure $(<140 /$ $90 \mathrm{mmHg}$ ) (Table 2). In addition, participants were recreationally active and free of diabetes and other metabolic diseases, and did not use any vasoactive medications. All women were postmenopausal and those using hormone replacement therapy were excluded.

\section{Screening visit}

The screening visit consisted of anthropometric measurements, determination of fasting blood lipids and glucose (Cholestech LDX System; Cholestech Corporation), and assessment of FMD (see below). Body mass was measured to the nearest $0 \cdot 1 \mathrm{~kg}$ on a calibrated digital scale and height $( \pm 0 \cdot 1 \mathrm{~cm})$ was measured using a stadiometer. Waist and hip circumference was determined and recorded to the nearest $0 \cdot 1 \mathrm{~cm}$. Following

Table 2. Subject characteristics ( $n$ 21, eleven males)

(Mean values with their standard errors)

\begin{tabular}{lrc}
\hline Variables & Mean & SEM \\
\hline Age (years) & $55 \cdot 2$ & $1 \cdot 3$ \\
Height $(\mathrm{cm})$ & $171 \cdot 6$ & $2 \cdot 7$ \\
Weight $(\mathrm{kg})$ & $82 \cdot 4$ & $3 \cdot 2$ \\
BMl $\left(\mathrm{kg} / \mathrm{m}^{2}\right)$ & $27 \cdot 8$ & $0 \cdot 6$ \\
Waist circumference $(\mathrm{cm})$ & $88 \cdot 1$ & $2 \cdot 5$ \\
Hip circumference $(\mathrm{cm})$ & $104 \cdot 9$ & $1 \cdot 7$ \\
SBP $(\mathrm{mmHg})$ & $124 \cdot 0$ & $2 \cdot 5$ \\
DBP $(\mathrm{mmHg})$ & $80 \cdot 0$ & $1 \cdot 8$ \\
HR (bpm) & $61 \cdot 0$ & $1 \cdot 9$ \\
TC (mg/l) & 2008 & 55 \\
TAG (mg/l) & 1122 & 131 \\
HDL (mg/l) & 514 & 28 \\
LDL (mg/l) & 1278 & 54 \\
Glucose (mg/l) & 998 & 22 \\
\hline
\end{tabular}

SBP, systolic blood pressure; DBP, diastolic blood pressure; HR, heart rate; TC, total cholesterol. 
a $15 \mathrm{~min}$ rest period in a supine position, blood pressure was assessed twice by auscultation and heart rate assessed by palpation.

\section{Flow-mediated dilation}

Participants were enrolled on the basis of resting FMD responses of $55.0 \%$ in order to identify individuals at a higher risk of having a cardiovascular event ${ }^{(22)}$. Brachial artery FMD was assessed by high-frequency ultrasonographic imaging. All tests were performed in a quiet, temperature-controlled room after a $20 \mathrm{~min}$ period in a supine position. The non-dominant arm was extended and positioned perpendicularly from the torso at the level of the heart. A rapid inflation and deflation pneumatic cuff was placed on the forearm immediately distal to the olecranon process to provide a stimulus to forearm ischaemia ${ }^{\text {(23) }}$. Using a 5-12 MHz multi-frequency linear array transducer attached to a high-resolution ultrasound machine (T3000; Terason), the brachial artery was imaged longitudinally in the distal third of the upper arm with clear visualisation of the anterior and posterior vessel walls. Simultaneous assessment of continuous Doppler velocity using the ultrasound machine was performed at an insonation angle of $60^{\circ}$. After optimisation of the image and blood velocity signal, the probe was held stable and enddiastolic baseline brachial artery images were recorded for $1 \mathrm{~min}$. The forearm cuff was then inflated to $200 \mathrm{mmHg}$ for 5 min using a rapid cuff inflator (Hokanson E20; D.E. Hokanson, Inc.) to occlude the brachial artery, and then released. Diameter and blood velocity recordings resumed $1 \mathrm{~min}$ before cuff deflation and continued for $3 \mathrm{~min}$ thereafter. Ultrasound images were recorded at a rate of five frames/s using screen capture software (Camtasia Studio; TechSmith Corporation) and converted into an AVI (audio video interleave) file. Anatomical measurements were made and internal landmarks identified to ensure placement of the transducer in the same location on the arm during subsequent measurements/visits. Off-line analyses of diameters and velocities were performed using automated edge-detection software with end-diastolic gating (Medical Imaging Applications). All vascular measurements and analyses were performed by the same technician.

Using a three-frame smoothing average, post-occlusion changes in diameter were determined. The peak postocclusion diameter was determined as the highest threeframe average. Relative (\%) and absolute ( $\mathrm{mm}$ ) brachial artery FMD was calculated from the preceding baseline diameter. From end-diastolic synchronised diameter $(D ; \mathrm{mm})$ and velocity data $(V ; \mathrm{m} / \mathrm{s})$, shear rate (an estimate of shear stress without viscosity; per $s$ ) was calculated as four times mean blood velocity per vessel diameter ${ }^{(24)}$. The shear rate stimulus responsible for endothelium-dependent FMD following cuff deflation was calculated in accordance with recent findings ${ }^{(25)}$. The area under the shear rate curve ( $\mathrm{SR}_{\mathrm{AUC}}$; arbitrary units), calculated for data up to the point of maximal post-occlusion diameter ${ }^{(25)}$, was determined to quantify the 'relevant' hyperaemic stimulus responsible for the peak FMD response. The relative peak FMD response was normalised to shear rate and expressed as the peak FMD:SR $\mathrm{AUC}$ ratio (arbitrary units).

\section{Acute vascular testing protocol}

The two acute trials occurred at the same time of the day separated by at least a 1 -week washout period. Following assessment of resting heart rate and blood pressure, fasting measurement of FMD was determined. Next, a flexible catheter was inserted into an antecubital vein and after a $15 \mathrm{~min}$ supine stabilisation period, blood samples were collected from a three-way stopcock connected to the end of the catheter for fasting baseline and subsequent postprandial biochemistry measurements. Immediately following these baseline measurements, subjects consumed a single prepackaged dose $(5 \mathrm{~g})$ of either NOP-47 with artificial sweetener or placebo mixed in water. NOP-47 is a proprietary extract isolated from a whey protein hydrolysate (Glanbia Nutritionals). The placebo was identical except the packets contained only artificial sweetener (aspartame and acesulfame potassium). Subjects ingested the beverage within $3 \mathrm{~min}$, after which a timer was started for the $2 \mathrm{~h}$ postprandial testing protocol. A questionnaire to address subjective symptoms and side effects associated with each supplement was administered at the end of the study.

Postprandial brachial artery FMD measurements were made at 30,60 and $120 \mathrm{~min}$, while blood samples were obtained at 15, 30, 60, 90 and $120 \mathrm{~min}$ post-ingestion. Blood pressure was assessed by the same investigator at 15, 45, 75, 105 and $125 \mathrm{~min}$ post-ingestion. Subjects remained supine in a comfortable position for the entire duration of the test.

Blood collection and biochemical analyses. Whole blood was collected into tubes with no additives (serum), or tubes containing EDTA, or lithium heparin and centrifuged $\left(1500 \mathrm{~g}, 15 \mathrm{~min}, 4^{\circ} \mathrm{C}\right)$. Before centrifugation, haematocrit was determined from the whole blood in duplicate using a microcapillary reader. Serum and plasma were transferred into storage tubes, snap frozen in liquid $\mathrm{N}_{2}$ and stored at $-80^{\circ} \mathrm{C}$ for future analysis. Samples for each assay were analysed in duplicate.

The liquid glucose hexokinase assay was used to measure plasma glucose in accordance with the manufacturer's instructions (Pointe Scientific, Inc.). Glucose concentrations were determined at $340 \mathrm{~nm}$ on a spectrophotometer (CV $4.5 \%$, VersaMax Microplate Reader; Molecular Devices Corporation). Insulin was measured from serum using a commercially available ELISA kit (CV 3.9\%, CalBiotech, Inc.) in accordance with the manufacturer's instructions.

Total nitrite/nitrate $\left(\mathrm{NO}_{x}\right.$ ), an index of $\mathrm{NO}^{\circ}$ production, was determined by the Griess reaction using a colorimetric method described by Giustarini et al. ${ }^{(26)}$ with minor modification (CV 3.0\%). Briefly, plasma samples were filtered using $10 \mathrm{kDa}$ molecular-weight cut-off filters before the analysis in order to reduce background absorbance. Diluted plasma filtrate (1:3) was mixed on a ninety-six-well plate with nitrate reductase (Sigma-Aldrich) and a solution containing NADPH and FAD, and then incubated for $90 \mathrm{~min}$ at room temperature. Following this, $200 \mu \mathrm{l}$ of Griess reagent were added to the wells and the plate was incubated for $30 \mathrm{~min}$ in the dark. Following incubation, samples were read at $540 \mathrm{~nm}$ on a microplate reader (SpectraMax M2; Molecular Devices 
Corporation). The major metabolite of the vasodilatory molecule prostacyclin, 6-keto PGF $_{1 \alpha}$ (CV 2.9\%; Cayman Chemical Company), and the potent vasoconstrictor endothelin-1 (ET-1, CV $4.1 \%$; R\&D Systems, Inc.) were measured in the plasma using commercially available ELISA kits. Serum ACE activity was determined before and at 30, 60 and 120 min post-ingestion using an enzymatic assay, with minor modification (CV $1.3 \%$; Alpco Immunoassays). Briefly, $250 \mu$ l of the synthetic substrate FAPGG were added to each well containing $25 \mu \mathrm{l}$ calibrator, control or unknown serum, and incubated at $37^{\circ} \mathrm{C}$ for $5 \mathrm{~min}$. The wavelength was then read twice at an interval of exactly $10 \mathrm{~min}$ at $340 \mathrm{~nm}$ to measure the decrease in the absorbance of the cleavage reaction mediated by ACE.

Plasma amino acids were analysed from blood samples obtained at baseline and at 30,60 and $120 \mathrm{~min}$ postprandial. Untreated $\mathrm{Na}$ heparin plasma samples were stored at $-80^{\circ} \mathrm{C}$ and processed using the protocol outlined below upon thawing at room temperature. The samples were initially centrifuged at $10000 \boldsymbol{g}$ for $5 \mathrm{~min}$ to remove any particulate matter from the fluid. Next, $25 \mu \mathrm{l}$ of the supernatant were diluted 4-fold with a $1.25 \mu \mathrm{m}$ internal standard solution of 3,5-diiodotyrosine. The resultant mixture was then transferred to a $3 \mathrm{kDa}$ molecular-weight cut-off filter (Pall Corporation) and centrifuged for $10 \mathrm{~min}$ at $7500 \mathrm{~g}$. The low-molecular-weight filtrate was then diluted $12 \cdot 5$-fold in the appropriate mobile phase and used for amino acid analysis.

Amino acids were quantified by ultra-performance liquid chromatography tandem MS (UPLC-MS/MS) using an Acquity Ultra-Performance LC system (Waters) coupled to a Waters Micromass ${ }^{\circledR}$ Quattro Premier triple quadruple instrument (Waters). The UPLC-MS/MS experimental parameters for determining underivatised amino acids have been detailed elsewhere $^{(27-29)}$. Data acquisition was performed using MassLynx $^{\mathrm{TM}} 4.1$ and QuanLynx ${ }^{\mathrm{TM}}$ software (Waters).

\section{Statistical analyses}

Statistical analyses were performed using STATISTICA (StatSoft, Inc.). All data were initially evaluated for homogeneity of variance and normality. Dependent variables that were not normally distributed were log transformed using the natural base to achieve normality. Untransformed values are reported with their significance levels because there were no qualitative differences in the results and they permit more meaningful interpretations than transformed data. Differences between the acute trials for dietary intakes and pre-ingestion values were evaluated using two-tailed Student's paired $t$ tests. Treatment and time effects for postprandial vascular and haematological responses were evaluated using twoway repeated-measures ANOVA with time points as the within effect and trial as the between effect. When main or interaction effects were present, Fisher's least significant difference post hoc test was used to assess pairwise comparisons. Multiple linear regression, controlling for within-subject repeated measures, was used to calculate correlation coefficients as described by Bland \& $\operatorname{Altman}^{(30)}$. The CV (within-subject SD/within-subject mean $\times 100$ ) for the baseline diameter, peak diameter and percentage of FMD (\%FMD) were $1.2,1.6$ and $32.2 \%$, respectively, and are comparable with previous investigations ${ }^{(31-33)}$. The $\alpha$-level for significance was set at $\leq 0 \cdot 05$.

\section{Results}

\section{Screening visit}

A total of twenty-one middle-aged adults (Table 2) qualified to participate in the two supplement trials. None of the variables assessed during the screening visit differed from either of the two supplement trials at baseline $(P>0 \cdot 05)$

\section{Acute ingestion trials}

No adverse events were reported by subjects during either trial. Additionally, no differences in dietary macronutrient composition were found between the trials (data not shown), indicating that participants maintained similar dietary patterns before each trial.

Vascular function. Pre-ingestion (PRE) FMD was similar between the trials $(P=0 \cdot 144$; Fig. 1(a)). A significant time and interaction effect for FMD $(P<0.05)$ indicated that FMD responses decreased from PRE at $60 \mathrm{~min}$ during the placebo trial. In addition, FMD responses during the NOP-47 trial increased at $30 \mathrm{~min}(P<0.05)$ and $120 \mathrm{~min}(P<0.001)$ relative to PRE. Moreover, FMD at $120 \mathrm{~min}$ was higher following NOP-47 ingestion than the corresponding placebo time point $(P<0 \cdot 05)$.

Pre-occlusion brachial artery diameters were not significantly different before ingestion of NOP-47 and placebo (Table 3; Fig. 1(b)). However, there was a significant main effect of time $(P=0.002)$, and post hoc analyses indicated that pre-occlusion brachial artery diameters decreased slightly from pre-ingestion at 30 and $120 \mathrm{~min}$ post-ingestion. No differences were detected between the trials $(P=0.52)$. In order to address the differences in pre-occlusion diameter within the trials, FMD was calculated as an absolute change. Similar to the above, a significant time $(P=0.020)$ and interaction effect $(P=0.054)$ for the absolute change in FMD indicated that FMD responses decreased $(P=0.031)$ from PRE $(0 \cdot 18$ $(\operatorname{sem} 0.02) \mathrm{mm})$ at $60 \mathrm{~min}(0.14(\operatorname{sem} 0.02) \mathrm{mm})$ during the placebo trial. Compared with PRE $(0 \cdot 16($ SEM $0 \cdot 02) \mathrm{mm})$, absolute FMD responses during the NOP-47 trial increased at $30 \mathrm{~min}(0.19$ (SEM 0.02$) \mathrm{mm} ; P=0.052)$ and $120 \mathrm{~min}(0.21$ (sem 0.02$) \mathrm{mm} ; P<0.01$ ). Moreover, the absolute change in FMD at 120 min was higher $(P=0.038)$ following NOP-47 ingestion than the corresponding placebo time point ( $0 \cdot 18$ (SEM $0.02) \mathrm{mm}$ and 0.21 (SEM 0.02$) \mathrm{mm}$ for $120 \mathrm{~min}$ placebo time point $v .120 \mathrm{~min}$ NOP-47 time point, respectively). No differences were detected between the trials for the absolute change in FMD $(P=0.36)$. There were no differences in peak brachial artery diameter over time or between the trials $(P>0 \cdot 05$; Table 3; Fig. 1(b)).

There was a main effect of time for pre-occlusion shear rate in that PRE had a significantly higher shear rate compared with 30 and 60 min post-ingestion for both trials $(P<0 \cdot 05$; Table 3$)$. The AUC of the post-occlusion shear rate calculated until the 
time-to-peak diameter measurement was significantly higher at PRE compared with the 30 and 60 min time points in both trials $(P<0.05$; Fig. 2). In addition, a significant main effect for trial $(P<0.05)$ was determined. However, post hoc analyses revealed no significant differences between the trials when data were collapsed $(P=0 \cdot 31)$. A significant main effect of time for mean arterial pressure was detected $(P<0 \cdot 05)$. Compared with PRE values (93.9 (SEM 2.0) $\mathrm{mmHg}$ ), mean arterial pressure increased $(P<0.05)$ slightly at $125 \mathrm{~min}$ post-ingestion (95.3 (sEM 1.9) $\mathrm{mmHg}$ ).

There was a main effect of time for FMD normalised to the magnitude of the shear rate stimulus (\%FMD/SR $\mathrm{AUC}_{\mathrm{AUC}} ; P<0.05$ ). Post hoc analyses indicated a trend for this ratio to increase at $30 \mathrm{~min}(P=0.069)$ and $120 \mathrm{~min}(P=0.065)$ post-ingestion, with no differences detected between the trials. There was no difference in the time-to-peak diameter over time or between the trials.

Haematological responses. Pre-ingestion biomarker concentrations did not significantly differ between the trials (Table 3). Compared with PRE, haematocrit increased slightly at 90 min post-ingestion but not at any other postprandial time point $(P<0 \cdot 01)$. Plasma glucose was unaffected by acute ingestion. Serum insulin increased significantly at $15 \mathrm{~min}$ $(P<0.01), 30 \mathrm{~min}(P<0.001)$ and $60 \mathrm{~min}(P<0.001)$ following NOP-47 ingestion (Fig. 3). The 15, 30 and $60 \mathrm{~min}$ insulin values were significantly higher following NOP-47 ingestion than the corresponding placebo time points $(P<0.01$ for time $\times$ trial interaction).

A significant main effect for trial $(P<0.05)$ and a trend for a time effect $(P=0.07)$ was determined for plasma $\mathrm{NO}_{x}$. When

(a)

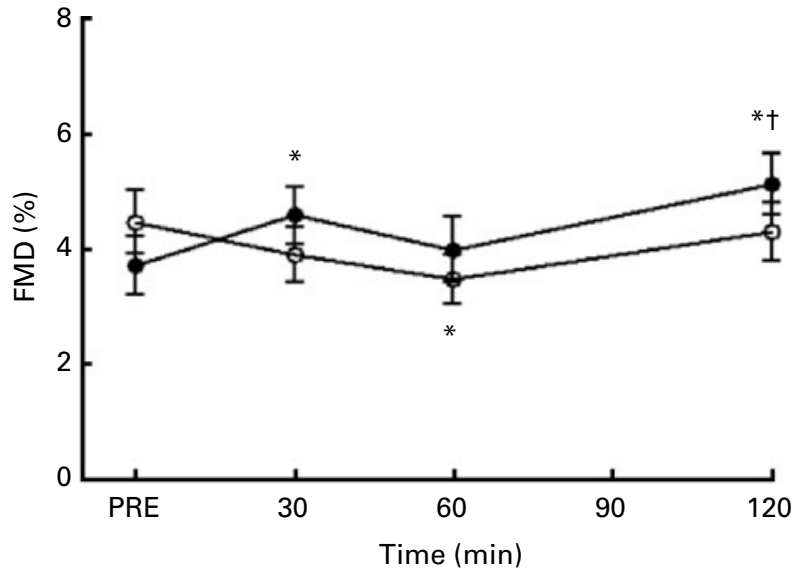

(b)

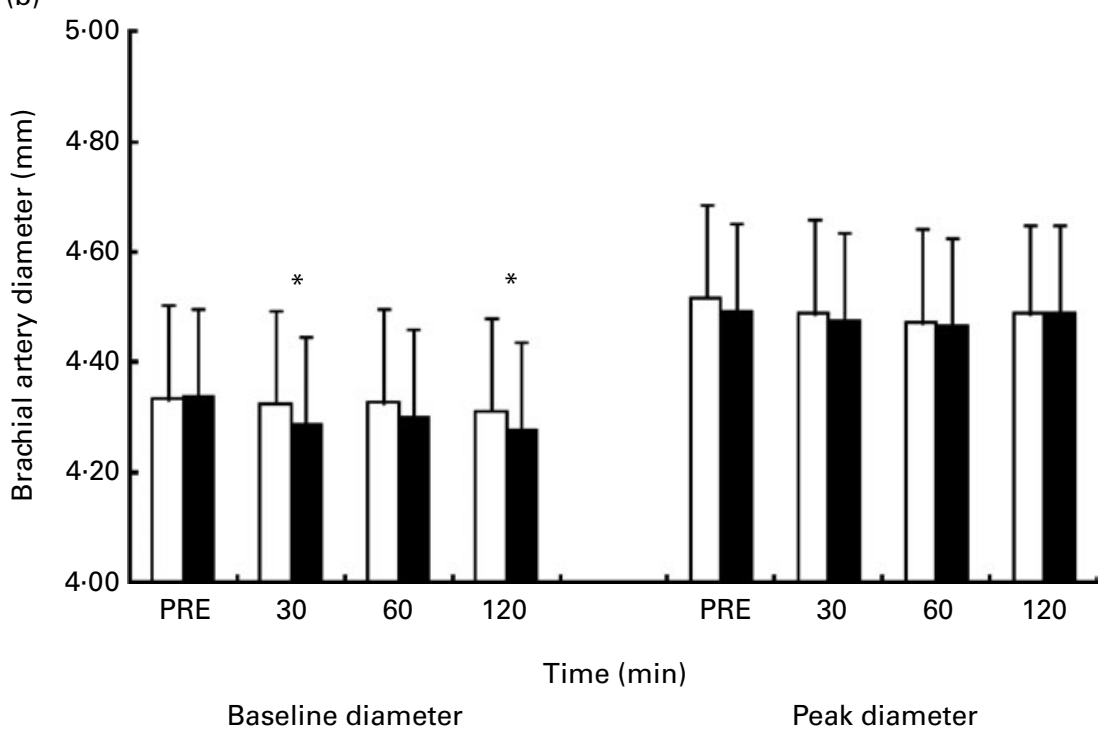

Fig. 1. Responses (a) for peak flow-mediated dilation (FMD) pre-ingestion (PRE) and following ingestion of placebo (O) or whey-derived extract (NOP-47; $\bullet$ ). Values are means, with standard errors represented by vertical bars. * Mean value was significantly different from that of PRE $(P<0.05$; Fisher's least significant difference (LSD) post hoc test). † Mean value was significantly different from that of the 120 min placebo time point $(P<0.05$; Fisher's LSD post hoc test). There were significant time $(P<0.05)$ and trial $\times$ time interaction $(P<0.05)$ effects. No effect was detected for trial $(P=0.43)$. (b) Baseline and peak brachial artery diameters PRE and following ingestion of placebo $(\square)$ or NOP-47 ( $\square$ ). Values are means, with their standard errors represented by vertical bars. ${ }^{*}$ Mean value was significantly different from that of PRE $(P<0.05$; Fisher's LSD post hoc test). There was a significant main effect of time $(P<0.01)$ for baseline diameter, but no effect for trial $(P=0.52)$. There was no significant trial $\times$ time interaction effect $(P=0.29)$. For peak diameter there was no significant effect for time $(P=0.18)$, or trial $(P=0.78)$ and no significant trial $\times$ time interaction effect $(P=0.89)$. 
concentrations were calculated as the change from PRE values, no main effects were detected (Fig. 4(a)).

Significant main effects for time $(P<0.05)$ and trial $(P=0.05)$ were detected for the major metabolite of prostacyclin, 6-keto $\mathrm{PGF}_{1 \alpha}$. Compared with PRE, 6-keto $\mathrm{PGF}_{1 \alpha}$ increased over time in both trials $(P<0.05)$ with higher values at 15 and $120 \mathrm{~min}$ post-ingestion $(P<0.05)$. When concentrations were calculated as the change from PRE values, no main effects were detected (Fig. 4(b)). Plasma levels of the potent vasoconstrictor ET-1 increased transiently at 15 and $30 \mathrm{~min}$ post-ingestion $(P<0.05)$ with no difference detected between the trials (Fig. 5). Serum ACE activity was unaffected in either trial $(P>0.05)$.
Pre-ingestion plasma amino acid concentrations were not different between NOP-47 and placebo. All concentrations of plasma amino acids, except for citrulline, increased after 30 min following ingestion of NOP-47 and returned to fasting levels within 60 or $120 \mathrm{~min}$ (Table 4). Plasma citrulline significantly decreased from PRE values at $30 \mathrm{~min}(P<0.0001)$, $60 \mathrm{~min}(P<0.0001)$ and $120 \mathrm{~min}(P<0.0001)$ post-ingestion for both trials. In general, the magnitude of the increase in plasma amino acids paralleled the amino acid composition of NOP-47 as exemplified by the 2-fold increase in plasma leucine at $30 \mathrm{~min}$. Concentrations of serine, threonine, asparagine, glutamic acid, valine, methionine, leucine, isoleucine, phenylalanine, tryptophan, glutamine, arginine, glycine,

Table 3. Vascular and haematological responses to acute ingestion of whey-derived extract (NOP-47) and placebo

(Mean values with their standard errors)

\begin{tabular}{|c|c|c|c|c|}
\hline \multirow[b]{2}{*}{ Variables } & \multicolumn{2}{|c|}{ NOP-47 } & \multicolumn{2}{|c|}{ Placebo } \\
\hline & Mean & SEM & Mean & SEM \\
\hline \multicolumn{5}{|c|}{ Pre-occlusion shear rate (per s) } \\
\hline Pre-ingestion & $171 \cdot 7$ & $13 \cdot 4$ & $158 \cdot 4$ & $10 \cdot 6$ \\
\hline $30 \min ^{*}$ & $152 \cdot 8$ & $13 \cdot 7$ & $129 \cdot 8$ & $9 \cdot 1$ \\
\hline $60 \min ^{*}$ & $146 \cdot 3$ & $14 \cdot 8$ & 133.9 & $9 \cdot 8$ \\
\hline $120 \mathrm{~min}$ & $158 \cdot 7$ & $12 \cdot 3$ & 141.4 & $11 \cdot 2$ \\
\hline \multicolumn{5}{|c|}{ Pre-occlusion brachial artery diameter ( $\mathrm{mm})$} \\
\hline Pre-ingestion & $4 \cdot 34$ & 0.16 & $4 \cdot 33$ & $0 \cdot 17$ \\
\hline $30 \mathrm{~min}^{*}$ & $4 \cdot 29$ & $0 \cdot 16$ & $4 \cdot 33$ & 0.17 \\
\hline $60 \mathrm{~min}$ & $4 \cdot 30$ & 0.16 & $4 \cdot 33$ & 0.17 \\
\hline $120 \min ^{*}$ & $4 \cdot 28$ & $0 \cdot 16$ & $4 \cdot 31$ & $0 \cdot 17$ \\
\hline \multicolumn{5}{|c|}{ Peak brachial artery diameter (mm) } \\
\hline Pre-ingestion & 4.49 & $0 \cdot 16$ & 4.52 & $0 \cdot 17$ \\
\hline $30 \mathrm{~min}$ & $4 \cdot 47$ & $0 \cdot 16$ & 4.49 & 0.17 \\
\hline $60 \min$ & 4.46 & 0.16 & 4.47 & 0.17 \\
\hline $120 \min$ & 4.49 & 0.16 & 4.49 & $0 \cdot 16$ \\
\hline \multicolumn{5}{|c|}{ Shear rate AUC (arbitrary units) } \\
\hline Pre-ingestion & $26473 \cdot 2$ & $2598 \cdot 7$ & $23773 \cdot 0$ & $2216 \cdot 2$ \\
\hline $30 \mathrm{~min}^{*}$ & $22025 \cdot 9$ & 2285.9 & $19308 \cdot 2$ & 1892.9 \\
\hline $60 \min ^{*}$ & $22329 \cdot 7$ & $1929 \cdot 8$ & $17543 \cdot 7$ & $1467 \cdot 8$ \\
\hline $120 \min$ & $24422 \cdot 4$ & $2198 \cdot 4$ & $22043 \cdot 3$ & $1589 \cdot 5$ \\
\hline \multicolumn{5}{|l|}{ TTP (s) } \\
\hline Pre-ingestion & $48 \cdot 0$ & $3 \cdot 6$ & $49 \cdot 2$ & $3 \cdot 3$ \\
\hline $30 \mathrm{~min}$ & $43 \cdot 2$ & $3 \cdot 4$ & $46 \cdot 5$ & 3.9 \\
\hline $60 \min$ & $49 \cdot 0$ & $4 \cdot 3$ & $41 \cdot 6$ & $3 \cdot 4$ \\
\hline $120 \mathrm{~min}$ & $49 \cdot 4$ & $4 \cdot 6$ & $47 \cdot 1$ & $4 \cdot 6$ \\
\hline \multicolumn{5}{|l|}{ Haematocrit (\%) } \\
\hline Pre-ingestion & $41 \cdot 0$ & 0.5 & $40 \cdot 2$ & 0.7 \\
\hline $15 \mathrm{~min}$ & $41 \cdot 1$ & 0.5 & $40 \cdot 6$ & 0.6 \\
\hline $30 \mathrm{~min}$ & $41 \cdot 3$ & 0.5 & $40 \cdot 4$ & 0.6 \\
\hline $60 \mathrm{~min}$ & $41 \cdot 0$ & 0.6 & $40 \cdot 4$ & 0.7 \\
\hline $90 \min ^{\star}$ & $41 \cdot 4$ & 0.6 & 40.9 & 0.6 \\
\hline $120 \min$ & $41 \cdot 1$ & 0.6 & $40 \cdot 6$ & 0.6 \\
\hline \multicolumn{5}{|c|}{ Plasma glucose (mg/l) } \\
\hline Pre-ingestion & 896 & 21 & 904 & 25 \\
\hline $15 \mathrm{~min}$ & 898 & 21 & 911 & 27 \\
\hline $30 \mathrm{~min}$ & 907 & 22 & 896 & 26 \\
\hline $60 \mathrm{~min}$ & 885 & 24 & 902 & 26 \\
\hline $90 \mathrm{~min}$ & 891 & 19 & 910 & 23 \\
\hline $120 \mathrm{~min}$ & 895 & 22 & 912 & 22 \\
\hline \multicolumn{5}{|c|}{ Serum ACE activity $(\mathrm{U} / \mathrm{I}) \dagger$} \\
\hline Pre-ingestion & $37 \cdot 5$ & $3 \cdot 3$ & $39 \cdot 0$ & $3 \cdot 8$ \\
\hline $30 \mathrm{~min}$ & $37 \cdot 4$ & 3.4 & 37.9 & $3 \cdot 7$ \\
\hline $60 \mathrm{~min}$ & $36 \cdot 3$ & $3 \cdot 8$ & $35 \cdot 3$ & 3.4 \\
\hline $120 \mathrm{~min}$ & $35 \cdot 0$ & $2 \cdot 6$ & $35 \cdot 8$ & $3 \cdot 0$ \\
\hline
\end{tabular}

AUC, area under the curve; TTP, time-to-peak diameter; ACE, angiotensin-converting enzyme.

* There was a significant main time effect $(P<0.05)$ from pre-ingestion.

$\dagger 1 \mathrm{U}=0.017 \mu \mathrm{kat}$. 


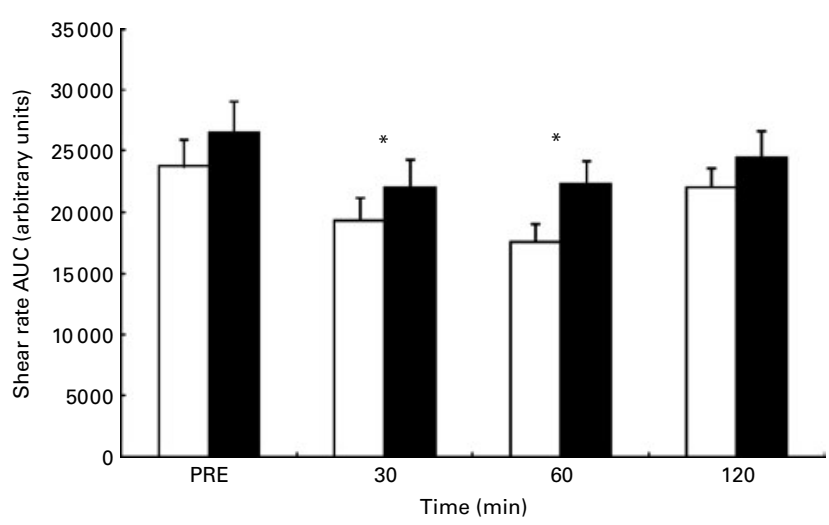

Fig. 2. Shear rate area under the curve (AUC) prior to pre-ingestion (PRE) and following ingestion of placebo $(\square)$ or whey-derived extract (NOP-47; $\square$ ). Values are means, with their standard errors represented by vertical bars. * Mean value was significantly different from that of PRE $(P<0.05$; Fisher's least significant difference post hoc test). There were significant effects for time $(P<0.01)$ and trial $(P=0.048)$ but no significant trial $\times$ time interaction effect $(P=0.784)$.

aspartic acid, citrulline, alanine, proline, ornithine and cysteine showed significant interaction effects $(P<0 \cdot 05$, time $\times$ trial), indicating that ingestion of NOP-47, but not placebo, resulted in a rapid increase in plasma amino acid levels.

Multiple linear regression, controlling for within-subject repeated measures, did not indicate a relationship between FMD and any haematological measure.

\section{Discussion}

The findings of the present study indicate that acute ingestion of an extract derived from whey protein was rapidly absorbed and improved endothelium-dependent dilation in older adults with vascular endothelial dysfunction. Impaired vascular endothelial function has been reported in healthy, sedentary middle-aged and older adults ${ }^{(11,12)}$. Therefore, we sought to determine the acute vascular and haematological effects of ingestion of a novel extract derived from whey in a population at risk for CVD. Similar to previous data from our laboratory in young healthy men and women ${ }^{(20)}$, acute ingestion of NOP-47 significantly increased postprandial FMD measured at 30 and 120 min post-ingestion in this group of participants at increased risk for CVD. The clinical significance of the transient changes in FMD in the present study of the order of $1-1.5 \%$ is uncertain. In asymptomatic low-risk individuals, lower FMD levels have been shown to predict future adverse cardiovascular events (a hazard ratio of 1.12 for every $1 \%$ decrease in FMD) ${ }^{(22)}$. Taking these findings into account, the transient changes in FMD seen in the present study would be viewed as significantly altering cardiovascular risk status and clinically relevant. The increase in FMD after NOP-47 ingestion was independent of changes in circulating metabolites of $\mathrm{NO}^{\circ}$, prostacyclin and ET-1, as these biomarkers were not different between the NOP-47 and placebo trials. However, local changes produced by the whey extract in vascular endothelium were not evaluated; therefore, the mediatory role of $\mathrm{NO}^{\circ}$, prostacyclin and $\mathrm{ET}-1$ in the observed transient increase of FMD cannot be excluded. As ageing is associated with enhanced oxidative stress and inflammation $^{(34,35)}$, it cannot be ruled out that NOP-47 ingestion transiently augmented FMD by attenuating elevated levels of oxidative stress and/or inflammation. However, previous research from our laboratory ${ }^{(20)}$ demonstrated that ingestion of NOP-47 enhanced FMD in the context of minimal changes in glucose and markers of oxidative stress, inflammation and antioxidant status in healthy young adults, making it unlikely that changes in oxidative stress contributed to the increase in FMD following NOP-47 ingestion in the present investigation.

In the placebo trial, we found a small but significant decrease in peak FMD at 60 min post-ingestion compared with baseline (mean absolute reduction $1.0 \%$ ), an effect not seen at the same time point following NOP-47 ingestion. The shorter time allowed between the 30 and $60 \mathrm{~min}$ FMD tests may explain this transient impairment. The present findings are in contrast to previous reports demonstrating no impairment in FMD when multiple FMD tests were performed closely together ${ }^{(33,36)}$. As reductions in the bioavailability of $\mathrm{NO}^{\circ}$ and increases in oxidative stress and inflammation play important roles in age-related endothelial dysfunction ${ }^{(34,35)}$, perhaps the older age of the participants studied in the present investigation accounts for our findings of slightly decreased FMD at $60 \mathrm{~min}$ following placebo ingestion. These findings might suggest that aged arteries are more vulnerable to ischae$\mathrm{mia} /$ reperfusion due to increased levels of oxidative stress and inflammation. Utilising a longer period of forearm ischaemia (20 min), Devan et al. ${ }^{(37)}$ demonstrated that sedentary ageing is associated with a greater magnitude of FMD impairment and delayed recovery from ischaemia/reperfusion compared with younger and age-matched physically active individuals. We are not aware of any investigations that have examined the impact of repetitive reactive hyperaemia on FMD assessed

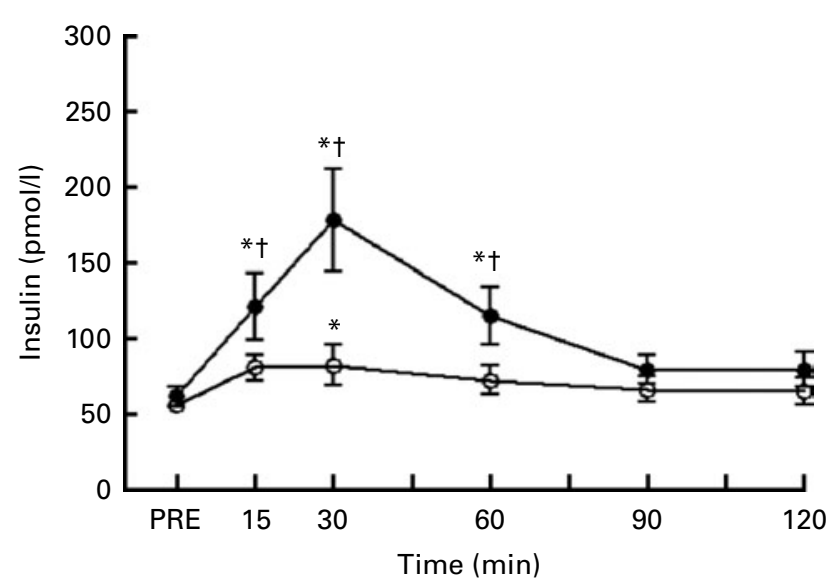

Fig. 3. Postprandial serum insulin responses to ingestion of a whey-derived extract (NOP-47; •) or placebo $(O)$. Values are means, with their standard errors represented by vertical bars. * Mean value was significantly different from that for pre-ingestion (PRE) $(P<0.05$; Fisher's least significant difference (LSD) post hoc test). † Mean value was significantly different from that for placebo at the same time point $(P<0.01$; Fisher's LSD post hoc test). There were significant trial $(P<0.01)$, time $(P<0.001)$ and trial $\times$ time interaction $(P<0.001)$ effects. 

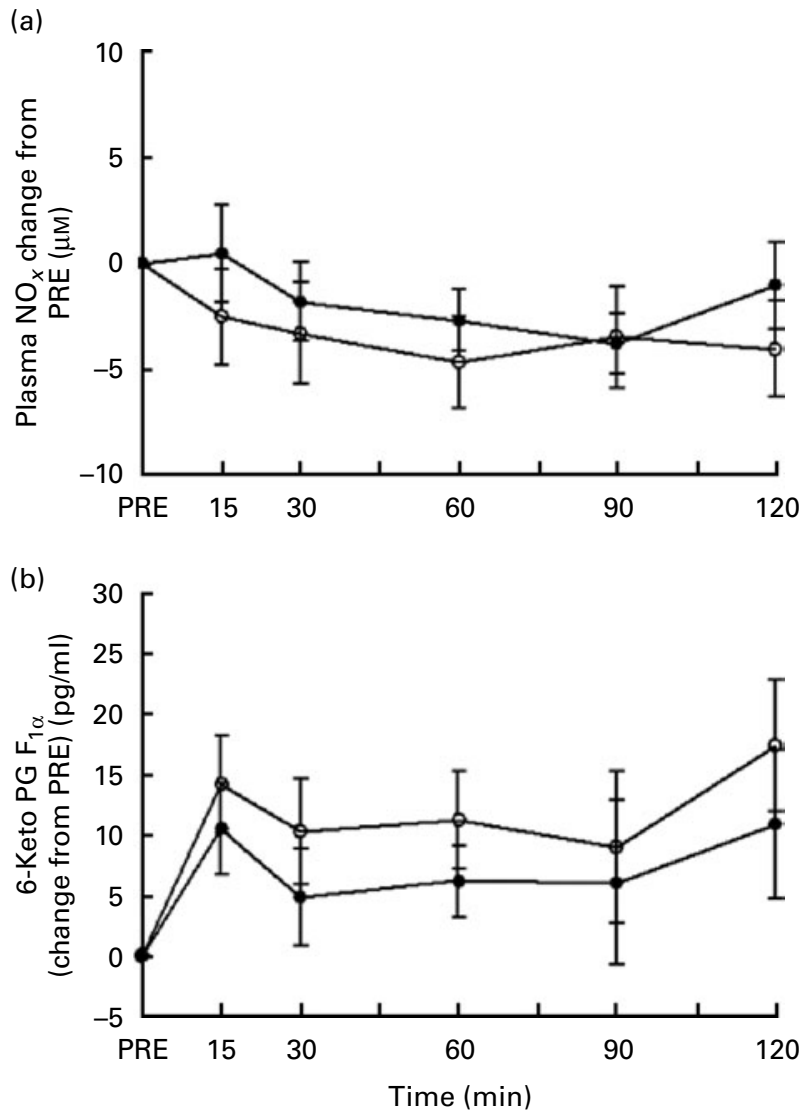

Fig. 4. (a) Plasma total nitrites/nitrates $\left(\mathrm{NO}_{x}\right)$ and (b) 6-keto $\mathrm{PGF}_{1 \alpha}$, normalised to pre-ingestion (PRE), responses to ingestion of a whey-derived extract (NOP-47; $\bullet$ ) or placebo $(O)$. Values are means, with their standard errors represented by vertical bars. For plasma $\mathrm{NO}_{x}$, there was no significant effect for trial $(P=0.49)$ or time $(P=0.24)$ and no significant trial $\times$ time interaction effect $(P=0.43)$ when concentrations were calculated as the change from PRE values. For 6 -keto $P F_{1 \alpha}$, there was no significant effect for trial $(P=0.10)$ or time $(P=0.27)$ and no significant trial $\times$ time interaction effect $(P=0.77)$ when concentrations were calculated as the change from PRE values.

following $5 \mathrm{~min}$ of ischaemia in older adults with an increased cardiovascular risk profile.

The results of the present study are in agreement with those of Nerla et al. ${ }^{(38)}$ and support the finding that two consecutive reactive hyperaemia-induced increases in shear stress performed in close succession transiently impairs FMD. In the study by Nerla et al., the reduction in FMD was associated with increased levels of the endogenous NO synthase inhibitor asymmetric dimethylarginine. Interestingly, when L-arginine was infused intravenously for $20 \mathrm{~min}$ before the two consecutive FMD measures, the impairment in the second FMD was prevented. These results suggest that $\mathrm{NO}^{\circ}$ depletion induced by increased levels of $\mathrm{NO}$ synthase inhibitors (i.e. asymmetric dimethylarginine) and/or reduced substrate availability impairs FMD when subsequent measurements are performed within close proximity. In the present study, NOP-47 ingestion prevented the decline in FMD at $60 \mathrm{~min}$, despite the extract being low in arginine $(<2 \%)$. Plasma concentrations of arginine increased transiently following the ingestion of NOP-47, but not placebo, demonstrating that increased substrate availability may be responsible for the preserved FMD seen at $60 \mathrm{~min}$ in the NOP-47 trial.

Compared with pre-ingestion, a significant decrease in the post-occlusion shear rate stimulus magnitude (i.e. $\mathrm{SR}_{\mathrm{AUC}}$ ) was detected at 30 and $60 \mathrm{~min}$ post-ingestion, with no differences between the trials. Similar results were obtained in young individuals who were subjected to four consecutive FMD tests separated by a mean inter-trial time of $13.5 \mathrm{~min}^{(33)}$. Despite this decreased stimulus, acute NOP-47, but not placebo, ingestion significantly increased FMD at $30 \mathrm{~min}$. The present data of a smaller shear rate stimulus in conjunction with an augmented FMD response would be interpreted as an increase in endothelial sensitivity to shear stress and improved endothelial function. Additionally, normalisation of the FMD response to the shear stress stimulus (\%FMD/SR $\mathrm{AUC}$ ) did not clarify our interpretation of increased FMD following NOP-47 ingestion. According to recent guidelines ${ }^{(39)}$, there must be at least a moderate correlation between FMD and the shear rate stimulus to justify normalisation of the response. Multiple linear regression, controlling for withinsubject repeated measures, indicated a weak correlation between \%FMD and shear rate AUC (combined trials: mean slope $0 \cdot 00004, r 0 \cdot 191, P=0 \cdot 020$ ), a finding that has previously been reported in older adults ${ }^{(40)}$.

Plasma insulin is elevated following L-arginine administration and may account for reported improvements in FMD following L-arginine supplementation ${ }^{(41,42)}$. Importantly, in the study by Gates et ll $^{(43)}$, the post-infusion FMD measurement ( $40 \mathrm{~min}$ post-infusion) was timed to isolate the effect of L-arginine from that of insulin, therefore reflecting an effect of L-arginine that is insulin-independent. In the present study, we measured FMD at 30,60 and 120 min post-ingestion with insulin returning to baseline levels within $90 \mathrm{~min}$. Therefore, the significantly greater FMD value detected at $120 \mathrm{~min}$ following NOP-47 ingestion suggests an insulin-independent mechanism.

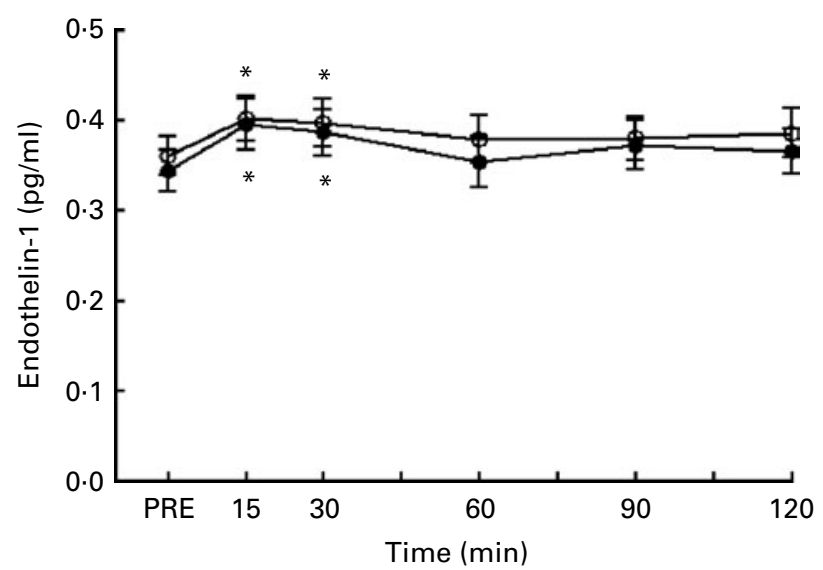

Fig. 5. Plasma endothelin-1 responses to ingestion of a whey extract (NOP47 ; ) or placebo $(\bigcirc)$. Values are means, with their standard errors represented by vertical bars. * Mean value was significantly different from that for pre-ingestion (PRE) $(P<0.05)$. There was a significant effect for time $(P<0.05)$ but no significant trial $(P=0.26)$ or trial $\times$ time interaction $(P<0.88)$ effect. 
Table 4. Fold increase in plasma amino acid concentrations that were significant due to the ingestion of a whey-derived extract (NOP-47)

\begin{tabular}{lcccccc}
\hline Amino acids & Pre-ingestion & $30 \mathrm{~min}$ & $60 \mathrm{~min}$ & $120 \mathrm{~min}$ & Time & Interaction \\
\hline Ser & 1.00 & 1.21 & 1.14 & 1.02 & 0.000 & 0.000 \\
Thr & 1.00 & 1.34 & 1.30 & 1.13 & 0.000 & 0.000 \\
Asn & 1.00 & 1.20 & 1.14 & 0.03 & 0.000 & 0.000 \\
Glu & 1.00 & 1.23 & 1.18 & 1.03 & 0.100 & 0.007 \\
Val & 1.00 & 1.33 & 1.27 & 1.06 & 0.000 & 0.000 \\
Met & 1.00 & 1.91 & 1.67 & 1.27 & 0.000 & 0.000 \\
Leu & 1.00 & 2.07 & 1.85 & 1.33 & 0.000 & 0.000 \\
Ile & 1.00 & 1.72 & 1.49 & 1.03 & 0.000 & 0.000 \\
Phe & 1.00 & 1.30 & 1.19 & 1.03 & 0.000 & 0.000 \\
Trp & 1.00 & 1.16 & 1.12 & 0.95 & 0.000 & 0.000 \\
Gln & 1.00 & 1.12 & 1.15 & 1.07 & 0.000 & 0.009 \\
Arg & 1.00 & 1.16 & 1.13 & 1.05 & 0.000 & 0.000 \\
Gly & 1.00 & 1.04 & 1.01 & 0.99 & 0.023 & 0.003 \\
Asp & 1.00 & 1.42 & 1.13 & 1.02 & 0.004 & 0.010 \\
Cit & 1.00 & 0.92 & 0.80 & 0.92 & 0.000 & 0.000 \\
Ala & 1.00 & 1.14 & 1.14 & 1.03 & 0.000 & 0.000 \\
Pro & 1.00 & 1.10 & 1.08 & 0.99 & 0.000 & 0.000 \\
Orn & 1.00 & 1.08 & 1.06 & 1.03 & 0.004 & 0.000 \\
Cys & 1.00 & 1.03 & 1.02 & 0.97 & 0.000 & 0.040 \\
\hline
\end{tabular}

To define the NOP-47-mediated activity on vasodilation, we measured $\mathrm{NO}^{\circ}$ status by evaluating total nitrite and nitrate levels $\left(\mathrm{NO}_{x}\right)$, the stable metabolites of $\mathrm{NO}^{\circ}$. When concentrations were calculated as the change from pre-ingestion values, no main effects were detected, indicating the absence of an effect of NOP-47 ingestion on circulating metabolites of $\mathrm{NO}^{\circ}$ in these middle-aged individuals (Fig. 4(a)). Although $\mathrm{NO}_{x}$ correlates with FMD, the assay is not specific for endothelial $\mathrm{NO}^{\circ}$ production and could also reflect $\mathrm{NO}^{\circ}$ derived from neuronal and inducible $\mathrm{NO}^{\circ}$ synthase ${ }^{(44)}$. Alternatively, alterations in the balance between vasodilators (e.g. bradykinin, adenosine, vascular endothelial growth factor and prostacyclin) and vasoconstrictors (e.g. endothelin, prostanoids and angiotensin II) have been suggested to contribute to the FMD response $^{(45)}$.

In order for oral ingestion of whey peptides to exert hypotensive or other biological effects in vivo, it must be absorbed intact and be transported to the target tissue while escaping destruction from intestinal brush-border or serum peptidases. Indeed, a specific whey-derived tripeptide with known high, in vitro ACE-inhibitory activity ${ }^{(46)}$ has been demonstrated to be absorbed intact following oral ingestion with a plasma elimination half-life of approximately $30 \mathrm{~min}^{(47)}$. The present data indicate that following acute ingestion of the wheyderived extract, concentrations of several amino acids, including the branched-chain amino acids, showed significant time and interaction effects, with increases detected at $30 \mathrm{~min}$ and concentrations returning to baseline levels at 60 or $120 \mathrm{~min}$. In general, the magnitude of the increase in plasma amino acids paralleled the amino acid composition of NOP-47 (Table 1) as exemplified by the 2-fold increase in plasma leucine at $30 \mathrm{~min}$. These individual amino acid data indicate that NOP-47 is quickly digested resulting in a rapid release of amino acids into the circulation, which reflects the amino acid composition of the extract. However, it cannot be determined from these data if specific bioactive peptides are absorbed intact that may potentially explain our in vivo biological effects. It is currently unknown as to which components of whey proteins (i.e. individual whey protein, peptide fractions, amino acids or synergistic actions among them) mediate the physiological effects of whey.

A possible mechanism by which whey proteins might improve endothelial function is through ACE inhibition. Human clinical trials have shown improvement in endothelial function in patients taking ACE inhibitors (reviewed in Tousoulis et al. ${ }^{(48)}$ ), which could be the result of pleiotropic effects of ACE inhibitors on the vascular endothelium ${ }^{(49)}$. Both decreased production of the vasoconstrictor angiotensin II and inhibited degradation of the vasodilatory molecule bradykinin occur with ACE inhibition, shifting the endothelial phenotype towards vasodilation. Dairy milk proteins inhibit ACE activity, with dairy whey proteins, in particular, exerting an anti-hypertensive effect in vitro ${ }^{(17,18)}$. However, in the present study, we were unable to detect an impact of NOP-47 ingestion on ACE activity measured in serum obtained from healthy human subjects. These data suggest that the transient increase in FMD demonstrated in the present study was not mediated via the inhibition of ACE.

Compared with pre-ingestion, the major metabolite of prostacyclin $\left(\mathrm{PGI}_{2}\right)$, 6-keto $\mathrm{PGF}_{1 \alpha}$, increased over time in both trials with higher values detected at 15 and $120 \mathrm{~min}$ post-ingestion. When concentrations were calculated as the change from pre-ingestion values, no main effects were detected. The rate of $\mathrm{PGI}_{2}$, which is the principal vascular prostanoid produced in the human vasculature ${ }^{(50)}$, secretion into the circulation coupled with its short half-life indicate that $\mathrm{PGI}_{2}$ functions locally ${ }^{(51)}$. Venepuncture may cause the release of $\mathrm{PGI}_{2}$ which will artifactually increase the 6-keto $\mathrm{PGF}_{1 \alpha}$ concentration in the plasma ${ }^{(52)}$.

Plasma levels of the potent vasoconstrictor ET-1 increased transiently at 15 and $30 \mathrm{~min}$ in both trials. Insulin stimulates secretion of the vasoconstrictive polypeptide ET-1 via the activation of mitogen-activated protein kinase-dependent branch of insulin signalling pathways ${ }^{(53)}$. A previous study 
demonstrated that insulin at physiological concentrations did not alter plasma ET-1 in healthy individuals, but did stimulate its secretion from endothelial cells in vitro ${ }^{(54)}$. Since ET-1 mainly exerts paracrine and autocrine effects, serum concentrations may not completely reflect its bioactivity. The findings of the present study using multiple sampling during the postprandial period indicate that a transient increase in plasma concentrations of ET-1 does occur that is not related to increased insulin levels as plasma insulin increased to a greater extent following NOP-47 ingestion. Minor alterations in plasma volume in the present study induced by fluid ingestion and/or phlebotomy may possibly explain the small, transient increases detected in plasma ET-1 concentration, an effect demonstrated previously ${ }^{(55)}$.

There are several limitations to the present study that should be mentioned. First, subjects were screened for impaired FMD based upon values obtained from a previous investigation ${ }^{(22)}$. In the present study, participants were enrolled on the basis of resting FMD responses of $\leq 5.0 \%$. This cut-off is consistent with the work of Shimbo et al. ${ }^{(22)}$ indicating that low FMD responses $(<7.5 \%)$ predict cardiovascular events in asymptomatic, lower-risk individuals at 36 months follow-up. In contrast to the present study where FMD was elicited following temporary occlusion of the forearm, Shimbo et al. used a FMD protocol that involved occluding the upper arm. Studies have indicated that forearm occlusion yields lower FMD values of the order of $2 \cdot 5 \%^{(56,57)}$. Thus, we used a cut-off of $5 \%$. The inclusion of a younger, control group with preserved FMD responses, along with a comparison of additional endothelial function measures at baseline, would have further confirmed endothelial dysfunction in the subjects investigated. Endothelium-independent dilation was not assessed so we cannot rule out an effect of the extract on vascular smooth muscle cells, as shown in vitro ${ }^{(58)}$. Furthermore, even though no differences in dietary macronutrient composition were found between the trials, dietary intake of nitrate or nitrite was not controlled for which may have added variability to the present $\mathrm{NO}^{\circ}$ results ${ }^{(59)}$. Despite this, we did not observe differences in pre-ingestion $\mathrm{NO}_{x}$ concentrations between the trials in the present study. Finally, the use of an iso-nitrogenous placebo, such as intact whey protein, would have helped us to further determine whether the effects seen in the two studies were due to a bioactive peptide specific to NOP-47 or simply by stimulation of insulin secretion by ingestion of whey ${ }^{(60)}$. In support of an insulin-independent mechanism, insulin was not related to \%FMD in either trial as indicated by multiple regression $(P=0.768$ and $P=0.884$ for placebo and NOP-47, respectively). These data suggest that differences in insulin responses between the two trials were not related to our observed changes in FMD.

The results of the present randomised, cross-over study suggest that in older individuals with impaired endothelial function (i.e. low FMD), acute ingestion of an extract derived from whey protein transiently improves endotheliumdependent vasodilation. The increase in FMD after whey extract ingestion was independent of changes in insulin and circulating metabolites of $\mathrm{NO}^{\circ}$, prostacyclin and ET-1. The extract investigated in this small sample of healthy, older individuals could be of clinical value as a potential therapy for patients with vascular pathologies who are at an increased CVD risk. Furthermore, studies investigating the potential of whey-derived extracts to attenuate the transient vascular impairment commonly demonstrated during the postprandial period or to augment the vasodilatory response to acute exercise are warranted and would have practical implications. In addition, future research should determine whether the effects of ingestion of whey-derived extracts can be augmented with higher or repeated doses throughout the day. Blockade studies are needed to further elucidate the precise mechanism(s)/pathway(s) by which novel whey-derived extracts exert their effects on the cardiovascular system.

\section{Acknowledgements}

This study was supported by Glanbia Nutritionals. We thank Loren Ward for providing technical expertise on whey-derived extracts and critically reading the manuscript. Daniel Friedenreich, Catherine Saenz, Juan Carlos Aristizibal, Laura Kunces and Laura Cusack are thanked for their assistance with the study intervention. K. D. B. contributed to the study conception and design, acquisition of the data, analysis and interpretation of the data and drafting and revising of the manuscript. B. R. K., B. M. V., and E. M. assisted with the data acquisition and analysis. A. S., C. L., A. S. P. and M. S. K. performed the plasma amino acid analysis. R. S. B., R. L. S., C. M. M. and W. J. K. contributed to the study conception and design and analysis and interpretation of the data. J. S. V. contributed to the study conception and design, interpretation of the data and drafting and revising of the manuscript. All authors read and approved the final manuscript. The authors declare that there are no conflicts of interest.

\section{References}

1. Lloyd-Jones D, Adams RJ, Brown TM, et al. (2010) Heart disease and stroke statistics-2010 update: a report from the American Heart Association. Circulation 121, e46-e215.

2. Lakatta EG \& Levy D (2003) Arterial and cardiac aging: major shareholders in cardiovascular disease enterprises: Part I: aging arteries: a "set up" for vascular disease. Circulation 107, 139-146

3. Furchgott RF \& Vanhoutte PM (1989) Endothelium-derived relaxing and contracting factors. FASEB J 3, 2007-2018.

4. Glasser SP, Selwyn AP \& Ganz P (1996) Atherosclerosis: risk factors and the vascular endothelium. Am Heart $J$ 131, 379-384.

5. Celermajer DS, Sorensen KE, Gooch VM, et al. (1992) Noninvasive detection of endothelial dysfunction in children and adults at risk of atherosclerosis. Lancet 340, 1111-1115.

6. Green D (2005) Point: flow-mediated dilation does reflect nitric oxide-mediated endothelial function. I Appl Physiol 99, 1233-1234, (discussion 1237-1238).

7. Parker BA, Tschakovsky ME, Augeri AL, et al. (2011) Heterogenous vasodilator pathways underlie flow-mediated dilation in men and women. Am J Physiol Heart Circ Physiol 301, H1118-H1126.

8. Anderson TJ, Uehata A, Gerhard MD, et al. (1995) Close relation of endothelial function in the human coronary and peripheral circulations. J Am Coll Cardiol 26, 1235-1241. 
9. Celermajer DS, Sorensen KE, Bull C, et al. (1994) Endothelium-dependent dilation in the systemic arteries of asymptomatic subjects relates to coronary risk factors and their interaction. J Am Coll Cardiol 24, 1468-1474.

10. Widlansky ME, Gokce N, Keaney JF, et al. (2003) The clinical implications of endothelial dysfunction. J Am Coll Cardiol 42, 1149-1160.

11. Celermajer DS, Sorensen KE, Spiegelhalter DJ, et al. (1994) Aging is associated with endothelial dysfunction in healthy men years before the age-related decline in women. $J \mathrm{Am}$ Coll Cardiol 24, 471-476.

12. Donato AJ, Gano LB, Eskurza I, et al. (2009) Vascular endothelial dysfunction with aging: endothelin-1 and endothelial nitric oxide synthase. Am J Physiol Heart Circ Physiol 297, H425-H432.

13. Taddei S, Virdis A, Ghiadoni L, et al. (2001) Age-related reduction of $\mathrm{NO}$ availability and oxidative stress in humans. Hypertension 38, 274-279.

14. Erdmann K, Cheung BW \& Schroder H (2008) The possible roles of food-derived bioactive peptides in reducing the risk of cardiovascular disease. J Nutr Biochem 19, 643-654.

15. FitzGerald RJ, Murray BA \& Walsh DJ (2004) Hypotensive peptides from milk proteins. J Nutr 134, 980S-988S.

16. Hirota T, Ohki K, Kawagishi R, et al. (2007) Casein hydrolysate containing the antihypertensive tripeptides Val-Pro-Pro and Ile-Pro-Pro improves vascular endothelial function independent of blood pressure-lowering effects: contribution of the inhibitory action of angiotensin-converting enzyme Hypertens Res 30, 489-496.

17. FitzGerald RJ \& Meisel H (1999) Lactokinins: whey proteinderived ACE inhibitory peptides. Nabrung 43, 165-167.

18. Kawase M, Hashimoto H, Hosoda M, et al. (2000) Effect of administration of fermented milk containing whey protein concentrate to rats and healthy men on serum lipids and blood pressure. J Dairy Sci 83, 255-263.

19. Pal S \& Ellis V (2010) The chronic effects of whey proteins on blood pressure, vascular function, and inflammatory markers in overweight individuals. Obesity (Silver Spring) 18, 1354-1359.

20. Ballard KD, Bruno RS, Seip RL, et al. (2009) Acute ingestion of a novel whey-derived peptide improves vascular endothelial responses in healthy individuals: a randomized, placebo controlled trial. Nutr J 8, 34 .

21. Harris RA, Nishiyama SK, Wray DW, et al. (2010) Ultrasound assessment of flow-mediated dilation. Hypertension $\mathbf{5 5}$, 1075-1085.

22. Shimbo D, Grahame-Clarke C, Miyake Y, et al. (2007) The association between endothelial dysfunction and cardiovascular outcomes in a population-based multi-ethnic cohort. Atherosclerosis 192, 197-203.

23. Corretti MC, Anderson TJ, Benjamin EJ, et al. (2002) Guidelines for the ultrasound assessment of endothelial-dependent flow-mediated vasodilation of the brachial artery: a report of the International Brachial Artery Reactivity Task Force. J Am Coll Cardiol 39, 257-265.

24. Parker BA, Trehearn TL \& Meendering JR (2009) Pick your Poiseuille: normalizing the shear stimulus in studies of flow-mediated dilation. J Appl Physiol 107, 1357-1359.

25. Pyke KE \& Tschakovsky ME (2007) Peak vs. total reactive hyperemia: which determines the magnitude of flowmediated dilation? I Appl Physiol 102, 1510-1519.

26. Giustarini D, Rossi R, Milzani A, et al. (2008) Nitrite and nitrate measurement by Griess reagent in human plasma: evaluation of interferences and standardization. Methods Enzymol 440, 361-380.
27. Piraud M, Vianey-Saban C, Bourdin C, et al. (2005) A new reversed-phase liquid chromatographic/tandem mass spectrometric method for analysis of underivatised amino acids: evaluation for the diagnosis and the management of inherited disorders of amino acid metabolism. Rapid Commun Mass Spectrom 19, 3287-3297.

28. Piraud M, Vianey-Saban C, Petritis K, et al. (2005) Ion-pairing reversed-phase liquid chromatography/electrospray ionization mass spectrometric analysis of 76 underivatized amino acids of biological interest: a new tool for the diagnosis of inherited disorders of amino acid metabolism. Rapid Commun Mass Spectrom 19, 1587-1602.

29. Piraud M, Vianey-Saban C, Petritis K, et al. (2003) ESI-MS/MS analysis of underivatised amino acids: a new tool for the diagnosis of inherited disorders of amino acid metabolism. Fragmentation study of 79 molecules of biological interest in positive and negative ionisation mode. Rapid Commun Mass Spectrom 17, 1297-1311.

30. Bland JM \& Altman DG (1995) Calculating correlation coefficients with repeated observations: Part 1 - Correlation within subjects. BMJ 310, 446

31. De Roos NM, Bots ML, Schouten EG, et al. (2003) Withinsubject variability of flow-mediated vasodilation of the brachial artery in healthy men and women: implications for experimental studies. Ultrasound Med Biol 29, 401-406.

32. Donald AE, Halcox JP, Charakida M, et al. (2008) Methodological approaches to optimize reproducibility and power in clinical studies of flow-mediated dilation. I Am Coll Cardiol 51, 1959-1964

33. Pyke KE \& Jazuli F (2011) Impact of repeated increases in shear stress via reactive hyperaemia and handgrip exercise: no evidence of systematic changes in brachial artery FMD. Am J Physiol Heart Circ Physiol 300, H1078-H1089.

34. Csiszar A, Wang M, Lakatta EG, et al. (2008) Inflammation and endothelial dysfunction during aging: role of NF-kappaB. J Appl Physiol 105, 1333-1341.

35. Rodriguez-Manas L, El-Assar M, Vallejo S, et al. (2009) Endothelial dysfunction in aged humans is related with oxidative stress and vascular inflammation. Aging Cell 8, 226-238.

36. Harris RA, Padilla J, Rink LD, et al. (2006) Variability of flow-mediated dilation measurements with repetitive reactive hyperemia. Vasc Med 11, 1-6.

37. Devan AE, Umpierre D, Harrison ML, et al. (2011) Endothelial ischemia-reperfusion injury in humans: association with age and habitual exercise. Am J Physiol Heart Circ Physiol 300, H813-H819.

38. Nerla R, Di Monaco A, Sestito A, et al. (2011) Transient endothelial dysfunction following flow-mediated dilation assessment. Heart Vessels 26, 524-529.

39. Thijssen DH, Black MA, Pyke KE, et al. (2011) Assessment of flow-mediated dilation in humans: a methodological and physiological guideline. Am J Physiol Heart Circ Physiol 300, $\mathrm{H} 2-12$.

40. Thijssen DH, Bullens LM, van Bemmel MM, et al. (2009) Does arterial shear explain the magnitude of flow-mediated dilation? A comparison between young and older humans. Am I Physiol Heart Circ Physiol 296, H57-H64.

41. Giugliano D, Marfella R, Verrazzo G, et al. (1997) The vascular effects of L-arginine in humans. The role of endogenous insulin. J Clin Invest 99, 433-438.

42. Kurz S \& Harrison DG (1997) Insulin and the arginine paradox. J Clin Invest 99, 369-370.

43. Gates PE, Boucher ML, Silver AE, et al. (2007) Impaired flowmediated dilation with age is not explained by L-arginine bioavailability or endothelial asymmetric dimethylarginine protein expression. J Appl Physiol 102, 63-71. 
44. Ishibashi T, Yoshida J \& Nishio M (1999) Evaluation of $\mathrm{NO}_{x}$ in the cardiovascular system: relationship to NO-related compounds in vivo. Jpn J Pharmacol 81, 317-323.

45. Deanfield JE, Halcox JP \& Rabelink TJ (2007) Endothelial function and dysfunction: testing and clinical relevance. Circulation 115, 1285-1295.

46. Wu J, Aluko RE \& Nakai S (2006) Structural requirements of Angiotensin I-converting enzyme inhibitory peptides: quantitative structure-activity relationship study of di- and tripeptides. J Agric Food Chem 54, 732-738.

47. Foltz M, Meynen EE, Bianco V, et al. (2007) Angiotensin converting enzyme inhibitory peptides from a lactotripeptideenriched milk beverage are absorbed intact into the circulation. J Nutr 137, 953-958.

48. Tousoulis D, Antoniades C, Koumallos N, et al. (2006) Novel therapies targeting vascular endothelium. Endothelium 13, 411-421.

49. Faggiotto A \& Paoletti R (1999) State-of-the-Art lecture. Statins and blockers of the renin-angiotensin system: vascular protection beyond their primary mode of action. Hypertension 34, 987-996.

50. Nowak J \& Wennmalm A (1979) Human forearm and kidney conversion of arachidonic acid to prostaglandins. Acta Physiol Scand 106, 307-312.

51. Frolich JC (1984) Measurement of icosanoids. Report of the Group for Standardization of Methods in Icosanoid Research. Prostaglandins 27, 349-368.

52. Catella F, Nowak J \& Fitzgerald GA (1986) Measurement of renal and non-renal eicosanoid synthesis. Am J Med 81, $23-29$.
53. Potenza MA, Marasciulo FL, Chieppa DM, et al. (2005) Insulin resistance in spontaneously hypertensive rats is associated with endothelial dysfunction characterized by imbalance between NO and ET-1 production. Am J Physiol Heart Circ Physiol 289, H813-H822.

54. Metsarinne K, Saijonmaa O, Yki-Jarvinen H, et al. (1994) Insulin increases the release of endothelin in endothelial cell cultures in vitro but not in vivo. Metabolism 43, 878-882.

55. Serneri GG, Modesti PA, Cecioni I, et al. (1995) Plasma endothelin and renal endothelin are two distinct systems involved in volume homeostasis. Am $J$ Physiol 268, H1829-H1837.

56. Agewall S, Doughty RN, Bagg W, et al. (2001) Comparison of ultrasound assessment of flow-mediated dilatation in the radial and brachial artery with upper and forearm cuff positions. Clin Physiol 21, 9-14.

57. Bots ML, Westerink J, Rabelink TJ, et al. (2005) Assessment of flow-mediated vasodilatation (FMD) of the brachial artery: effects of technical aspects of the FMD measurement on the FMD response. Eur Heart J 26, 363-368.

58. Sipola M, Finckenberg P, Vapaatalo H, et al. (2002) Alphalactorphin and beta-lactorphin improve arterial function in spontaneously hypertensive rats. Life Sci 71, 1245-1253.

59. Wang J, Brown MA, Tam SH, et al. (1997) Effects of diet on measurement of nitric oxide metabolites. Clin Exp Pharmacol Physiol 24, 418-420.

60. Power O, Hallihan A \& Jakeman P (2009) Human insulinotropic response to oral ingestion of native and hydrolysed whey protein. Amino Acids 37, 333-339. 